\title{
Article Use and Its Relationship to Individual User Satisfaction
}

I

N LIBRARY RESEARCH concerned with the individual's use of journals, serials, or monographs, a careful definition of terms must be made relative to such use. For example, it is quite different to speak of "satisfying a percentage of user circulation requirements" than to speak of "satisfying a percentage of the users." In the former case, one is speaking of the total number of loans or transactions regardless of which user has made the request, and in the latter case one refers to circulation satisfaction of individuals.

For example, if a given library is satisfying 80 percent of its overall requests, this statistic does not mean that 80 percent of the individuals making these requests had their requests fully satisfied, nor does it mean that each user had 80 percent of his requests satisfied. The statistic simply means that 80 percent of the total number of requests were satisfied. The extent of satisfaction of individual user requests would depend upon the number and variety of requests from each individual. Some users might have all of their requests satisfied, others only some, and others, none at all. Thus one could speak of the individual's user requirements being fully satisfied, partially satisfied, or not satisfied.

User requests for Xerox copies of journal articles at the Air Force Cambridge Research Laboratory (AFCRL)

Dr. Trueswell is Head, Department of Industrial Engineering, at the University of Massachusetts. library provide a specific illustration. Information collected by Groos about library user requests was subdivided into four time spans (1960-1966, 1946-1959, 1900-1945, and 0-1899) according to the year of publication of the journal article. ${ }^{1}$ The percentage use, that is, percentage satisfaction by users of their total requests, varied according to which time span was considered. Also, for a given time span, the user satisfaction for an individual (expressed as a percentage of his total requests) could range from zero to 100 percent.

The Groos data cover all requests by the users of the AFCRL library for Xerox copies of articles in the subject area of astronomy and directly related journals (a total of fifty-seven journal titles). Records of Xerox copy requests have been maintained from 1964 to 1966 and include information on the requester, the bibliographic citation, and number of copies reproduced. The data from Groos on astronomy are used in this article as a workable subset of the population of Xerox requests at the AFCRL library. All statistics and discussions in this article refer only to this astronomy subset.

Figure I is plotted on the basis of year of publication of the article as a variable. There are three plots on Figure I: one (dotted line) refers to article request satisfaction and the other two (solid lines) refer to individual user satisfaction. Thus the plot of "article request satisfaction" in Figure I represents the percentage of the articles copied that were published after a given 


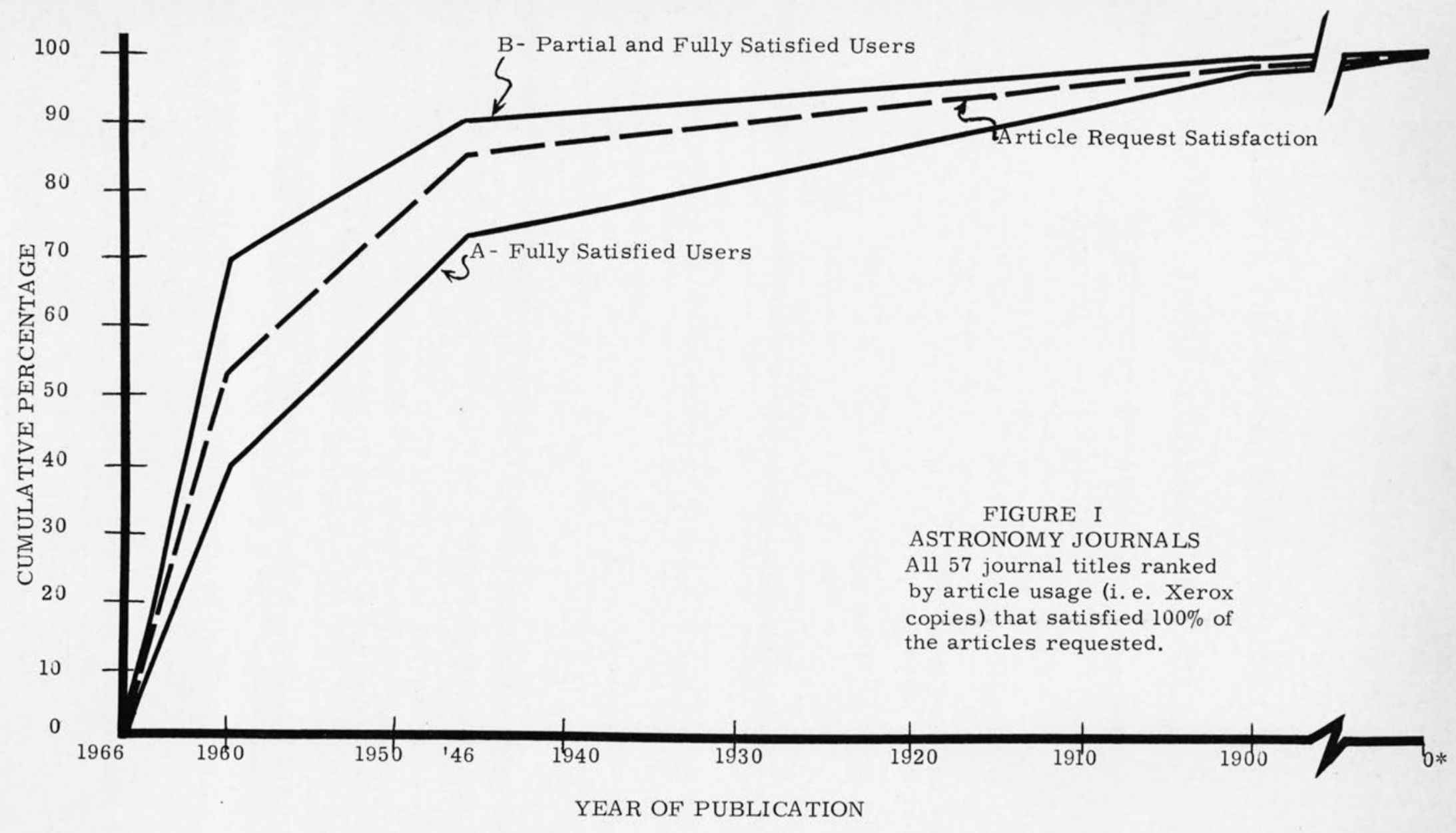

*First issue. 


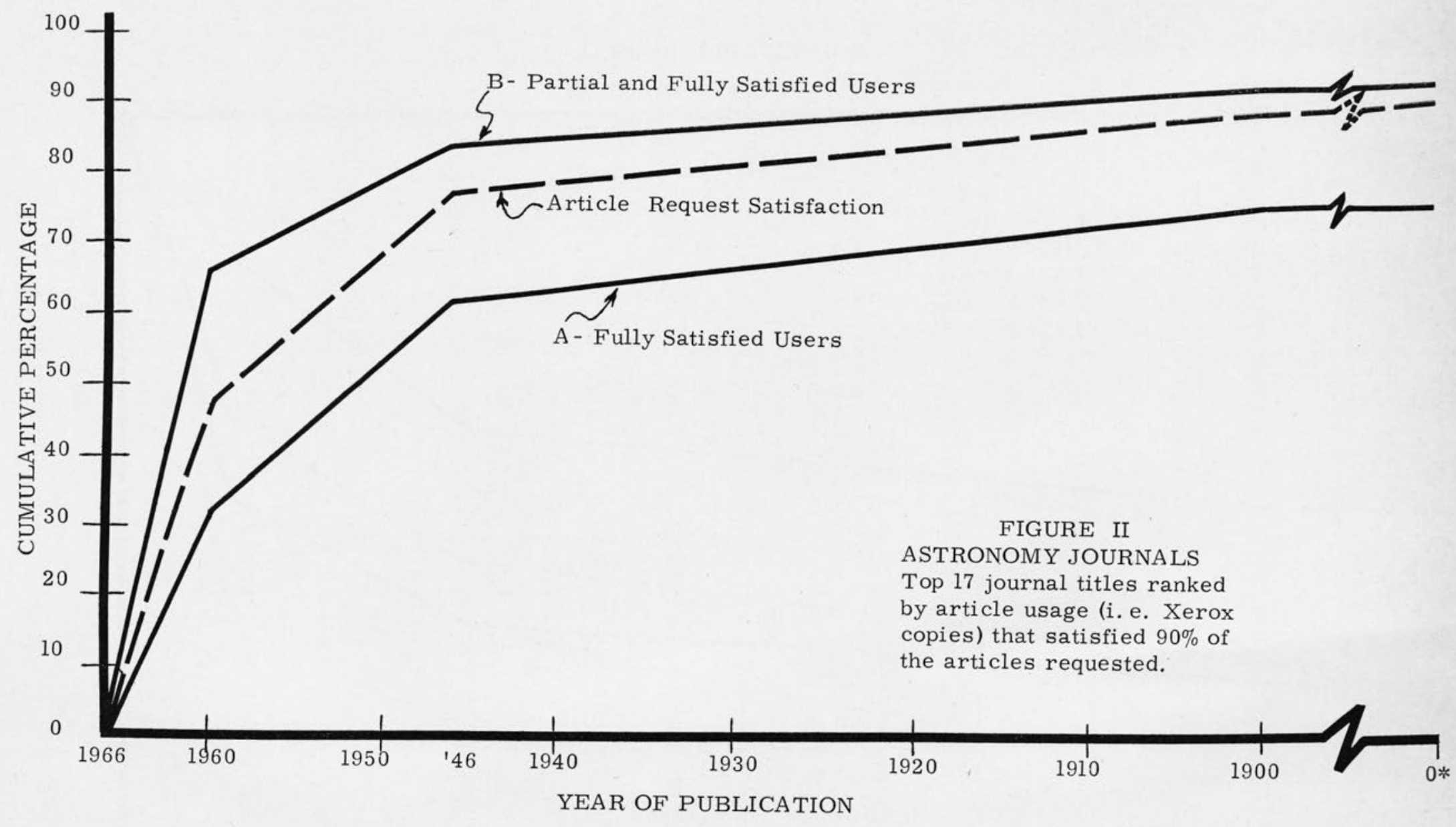

*First issue 


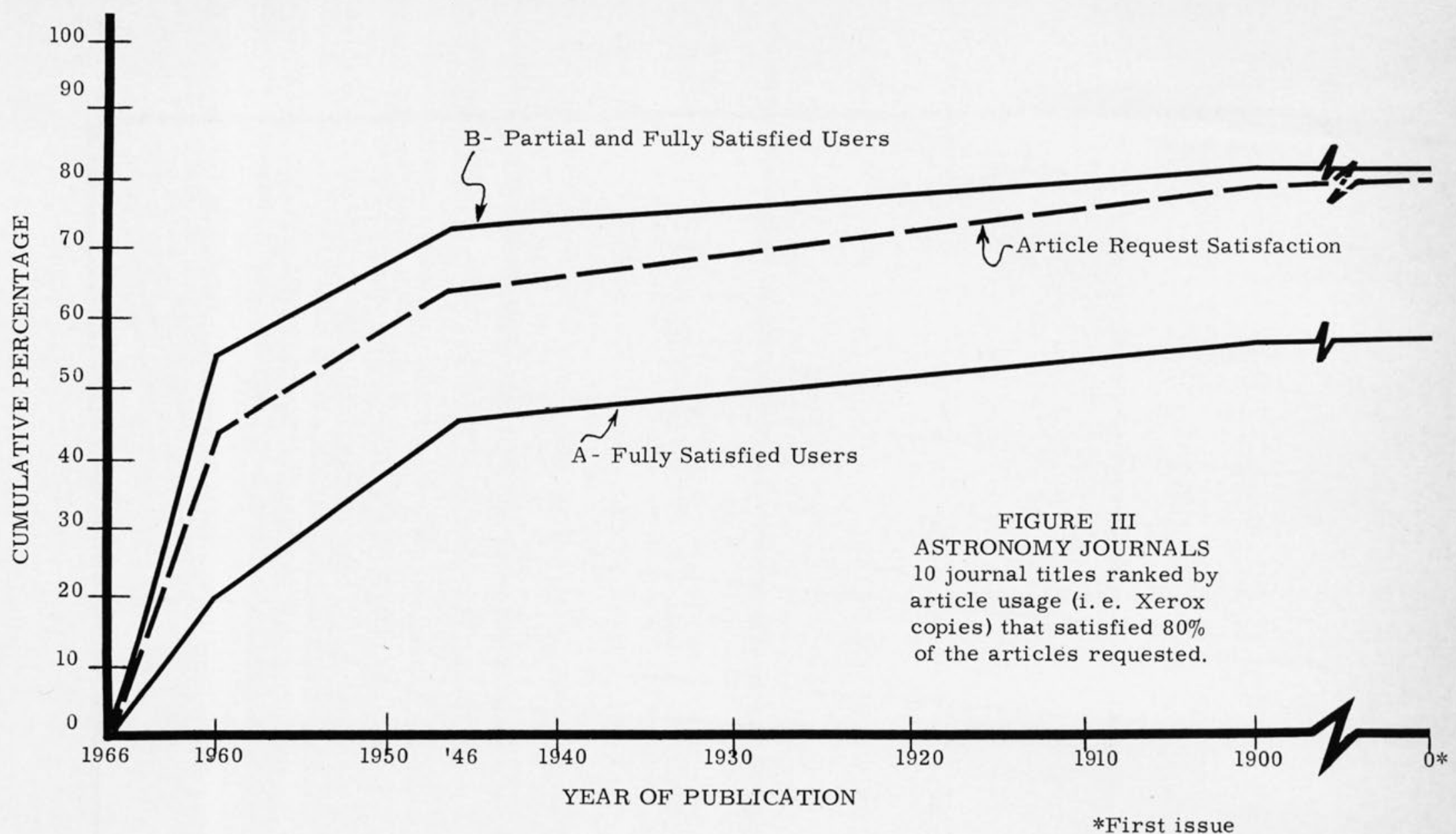




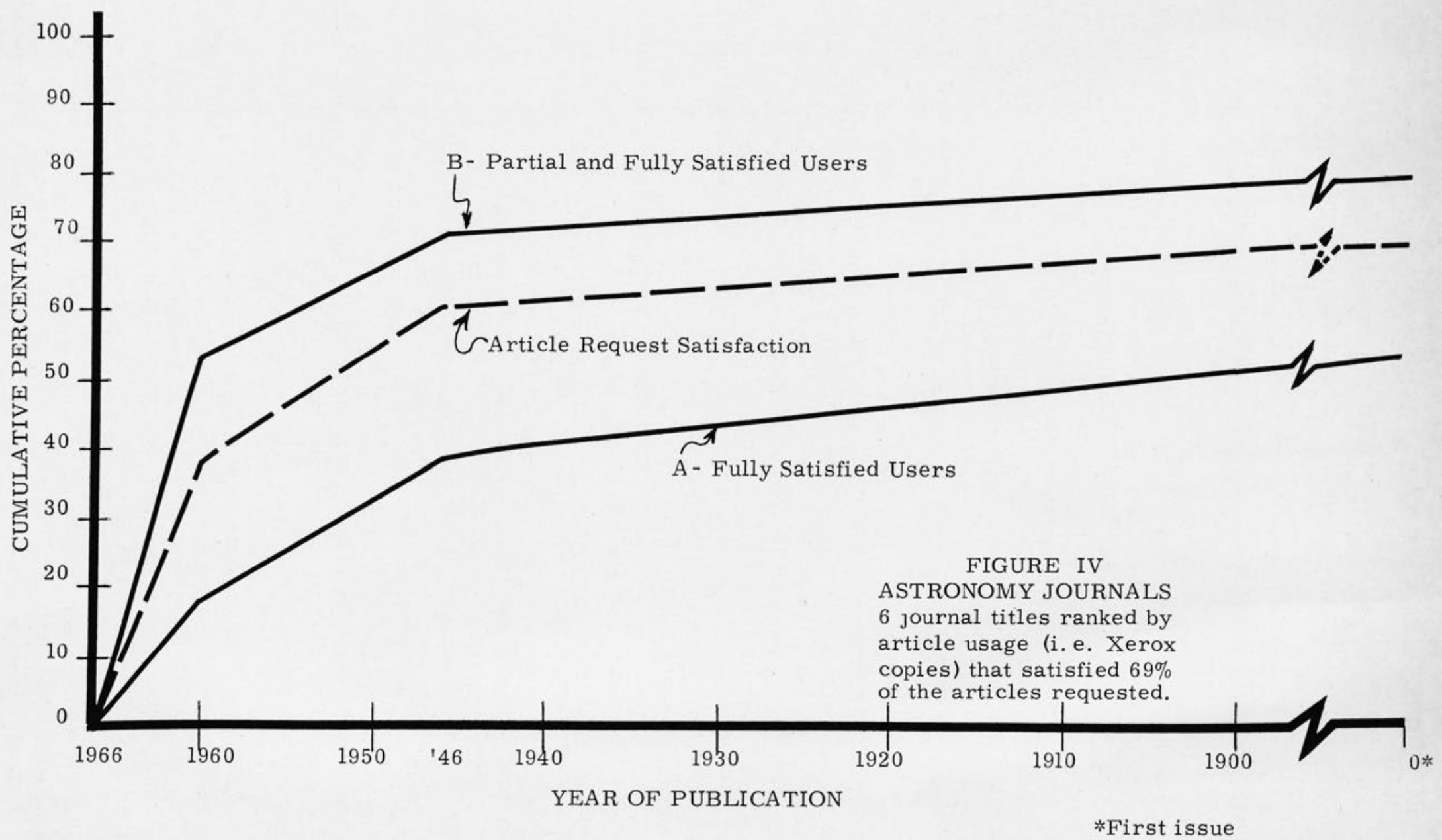


year. For example, approximately 85 percent of the articles copied were published after 1945.

However, the question remains as to what percentage of users will be fully satisfied by this time span (i.e., after 1945). This can be found from plot A in Figure I to be 73 percent (e.g., 73 percent of the users would have their requests fully satisfied by journals published after 1945). Similarly, 90 percent would have their requests partially or fully satisfied by journals published after 1945. Note that these figures essentially indicate that 10 percent of the total number of users (e.g., 100 percent minus 90 percent) have none of their requests satisfied, 17 percent (e.g., 90 percent minus 73 percent) are partially satisfied, and 73 percent are fully satisfied. Similar points have also been plotted on Figure I for 1900 and 1960. Note that straight lines have been drawn between the points because the exact shape of the curve is not known due to the method of partitioning of the original data.

It should be noted that the area between curves A and B on all four figures represents those users who would have their requests partially satisfied and the area above Curve B represents those users who do not have any of their requests satisfied. Similarly, the area below Curve A represents users who have all of their requests satisfied.

Thus as an illustration of the format of Figure I, the following statements can be made about the fifty-seven journal titles covered by these data, if for example, only the period 1960 and later is considered: (1) 40 percent of the users would have all of their requests satisfied, (2) 30 percent (i.e., 70 percent minus 40 percent) would have at least some part of their requests satisfied, (3) 30 percent (i.e., 100 percent minus 70 percent) would have none of their requests satisfied, and (4) 53 percent of all of the article requests would be satisfied. Although 53 percent of the articles copied are in the time span 1960-1966, there will still be 60 percent of the users having only some or none of their requests satisfied and only 40 percent having all of their requests satisfied.

Another way that the Xerox copy request data may be considered is to rank all fifty-seven of the astronomy journal titles according to articles copied/title. Thus, if only those journal titles that satisfy 90 percent of the articles copied are considered, we find that seventeen journals satisfy 90 percent of the article requests. The data for these seventeen journal titles are plotted on Figure II in the same manner as Figure I. We now have an indication of user satisfaction and article satisfaction vs. time for the top seventeen journal titles.

From Figure II we see the possible effect on user satisfaction over time of the decision to keep only those journals that would satisfy 90 percent of the article copy requests. We can also see the effect of considering two parameters jointly. For example, in Figure II we see that the top seventeen journals (ranked by articles/title) published after 1945 satisfied 77 percent of the total article requests, but only 61 percent of all of the users were fully satisfied.

Figures III and IV are an extension of the analysis that gave Figure II above. However, in Figures III and IV, only those journal titles are considered that give 80 percent and 69 percent article satisfaction, respectively. Here we see the effect on user satisfaction if we consider only the top ten (Figure III) or top six (Figure IV) journal titles out of a possible fifty-seven journal titles. Note that in Figures II through IV the combined effects of two variables may be considered, namely, the variable of subdivision of the journal titles by article usage and the variable of year of publication.

\section{Summary}

The above data helps to provide some 
insight into the relationship between overall article use and individual user satisfaction for journal articles in the subject area of astronomy. However, of perhaps greater interest is the strong indication that one should quantitatively consider both individual user satisfaction and overall article use before actually implementing any decision rules relating to the size of journal holdings in a library.

The librarian must decide how the statistics that pertain to article use and individual user satisfaction should be weighed in the determination of journal holdings. However, the use of these criteria is dependent upon the amount of data available from research in this area, which to date has been limited. The results, therefore, described in this article should not be taken as having general applicability to other disciplines or other library populations. A separate article use/user satisfaction relationship must be developed for each field.

\section{REFERENCE}

1. O. V. Groos, "Note to Meadows' Citation Characteristics of Astronomical Research Literature," Journal of Documentation, vol. 25, no. 4 (Dec. 1969). 